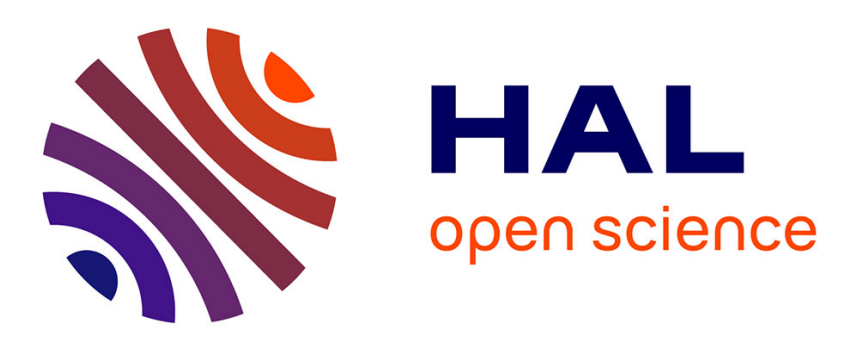

\title{
Spherical parameterization for genus zero surfaces using Laplace-Beltrami eigenfunctions
}

\author{
Julien Lefèvre, Guillaume Auzias
}

\section{To cite this version:}

Julien Lefèvre, Guillaume Auzias. Spherical parameterization for genus zero surfaces using LaplaceBeltrami eigenfunctions. Second International Conference, GSI 2015, Palaiseau, France, October 2830, 2015, Proceedings, Frank Nielsen Frederic Barbaresco, Oct 2015, Palaiseau, France. hal-01222933

\section{HAL Id: hal-01222933 \\ https://hal.science/hal-01222933}

Submitted on 31 Oct 2015

HAL is a multi-disciplinary open access archive for the deposit and dissemination of scientific research documents, whether they are published or not. The documents may come from teaching and research institutions in France or abroad, or from public or private research centers.
L'archive ouverte pluridisciplinaire HAL, est destinée au dépôt et à la diffusion de documents scientifiques de niveau recherche, publiés ou non, émanant des établissements d'enseignement et de recherche français ou étrangers, des laboratoires publics ou privés. 


\title{
Spherical parameterization for genus zero surfaces using Laplace-Beltrami eigenfunctions
}

\author{
Julien Lefèvre ${ }^{1,2}$, Guillaume Auzias ${ }^{1,2}$ * \\ 1 Aix-Marseille Université, LSIS, CNRS UMR 7296, Marseille, France \\ 2 Institut de Neurosciences de la Timone UMR 7289, Aix Marseille Université, \\ CNRS, Marseille, France \\ julien.lefevre@univ-amu.fr, guillaume.auzias@univ-amu.fr
}

\begin{abstract}
In this work, we propose a fast and simple approach to obtain a spherical parameterization of a certain class of closed surfaces without holes. Our approach relies on empirical findings that can be mathematically investigated, to a certain extent, by using Laplace-Beltrami Operator and associated geometrical tools. The mapping proposed here is defined by considering only the three first non-trivial eigenfunctions of the Laplace-Beltrami Operator. Our approach requires a topological condition on those eigenfunctions, whose nodal domains must be 2 . We show the efficiency of the approach through numerical experiments performed on cortical surface meshes.
\end{abstract}

Keywords: Riemannian Manifold, Laplace-Beltrami Operator, Surface Parameterization, Nodal Domains

\section{Introduction}

Spherical parametrization of 3D closed (genus-0) meshes is a classical approach in computer graphics for texture mapping, remeshing and morphing [11. Neuroimaging data analysis is an important field of applications since representing the brain as a closed surface is increasingly popular in the community, in line with the specific advantages for e.g. visualization and inter-subjects mapping [5]. Several works [7/1] translated most recent methodological advances from computer graphics to brain mapping, and put emphasis on two properties that are particularly desirable in this field [8]: the spherical parameterization must be fold-free to ensure the validity of neuroimaging data analysis that rely on the spherical representation, and computationally efficient in order to be applicable to large number of individual meshes whose sub-milimetric resolution involves typically more than $100 \mathrm{~K}$ vertices.

In this work, we propose a fast and simple approach to obtain a spherical parameterization of a certain class of genus 0 surfaces. Our approach is a particular case of the one defined by Bérard in [3], by considering only the three first non-trivial

\footnotetext{
* This work is funded by the Agence Nationale de la Recherche (ANR-12-JS03-001-01, "Modegy").
} 
eigenfunctions of the Laplace-Beltrami Operator. A comparable approach has also been proposed in $[8$ but implies to solve a highly non linear partial differential equation. We note also an empirical approach based on the spatial regularity of the first eigenfunctions in [10. We complement these papers with preliminary theoretical contributions, which echo with empirical findings that make it very appealing for neuroimaging studies. In particular, our contributions support the $C^{\infty}$ diffeomorphic nature of our mapping, under specific condition on the considered surface. We show through numerical experiments performed on 138 cortical surface meshes that this condition is systematically met in practice. Moreover, the resulting mapping is computed in few seconds and almost diffeomorphic with less than $1 \%$ of folded triangles on average.

\section{Background and main results}

We recall first classical results on Spectral theory and Laplace-Beltrami eigenfunctions. The interested reader can refer to [12].

\subsection{Laplace-Beltrami eigenfunctions}

Definition 1 Given $(\mathcal{M}, g)$ a compact 2-Riemannian manifold without boundary, denoting $x(p)=\left(x_{1}, x_{2}\right)$ a local coordinate system, i.e. a local diffeomorphism $\mathcal{M} \rightarrow \mathbb{R}^{2}$ around a point $p$, the Laplace-Beltrami operator acting on $C^{\infty}$ functions is

$$
\Delta f=\frac{1}{\sqrt{\operatorname{det}(g)}} \sum_{i, j} \partial_{x_{j}}\left(\sqrt{\operatorname{det}(g)} g^{i, j} \partial_{x_{i}} f\right)
$$

Remark 1. In the applications $\mathcal{M}$ will be a closed surface in $\mathbb{R}^{3}$ and the metric $g$ will correspond to the euclidean inner product.

If we consider the eigenvalue problem:

$$
\Delta f=-\lambda f
$$

we know that it has eigenvalues $0=\lambda_{0}<\lambda_{1} \leq \lambda_{2} \ldots$ and corresponding eigenfunctions $\Phi_{1}, \Phi_{2}, \ldots$ (See Fig 1 for a visual intuition of some $\Phi_{i}$ ). The eigenfunctions are orthogonal in the sense of the scalar product $\langle u, v\rangle_{\mathcal{M}}=\int_{\mathcal{M}} u v d \mu$, where the volume form $d \mu$ is given by $\sqrt{\operatorname{det}(g)} d x_{1} d x_{2}$.

Definition 2 Given an eigenfunction $\Phi$ of the Laplace-Beltrami Operator, we call nodal set the set of points where $\Phi$ vanishes. We denote it $N(\Phi)$ in the following. The nodal domains correspond to the connected components of the complementary of the nodal set.

We have some qualitative results on the nodal domains of eigenfunctions: 
Theorem 1 (Courant's nodal domain theorem) The number of nodal domains for the $n$-th eigenfunction is inferior or equal to $n+1$.

There is a global result on the dimension of nodal sets when $\mathcal{M}$ is a 2-manifold:

Theorem 2 (S.H. Cheng [4]) Except on a closed set of points, the nodal set of an eigenfunction $\Phi$ is a $C^{\infty} 1$-manifold, i.e. a line in our applications.

Last we recall the Green formula for an open set $D \in \mathcal{M}$ that will be of great use in the following:

$$
\int_{D} \Phi \Delta \Psi d \mu=-\int_{D} g(\nabla \Phi, \nabla \Psi) d \mu+\int_{\partial D} \Phi(\nabla \Psi \cdot n) d \tilde{\mu}
$$

$d \tilde{\mu}$ is the induced metric on the boundary. For simplicity, we use the notation - instead of the riemannian metric $g(.,$.$) and remove the volume forms in the$ following.
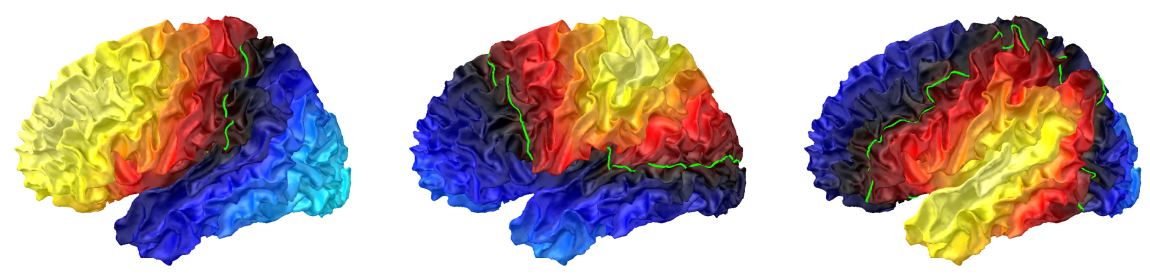

Fig. 1: From left to right: eigenfunctions $\Phi_{1}, \Phi_{2}$ and $\Phi_{3}$. Colormap goes from blue (negative) to red/yellow (positive). Each nodal sets are in green.

\subsection{Main conjecture}

Based on empirical findings we suggest the following result, which allows to define a natural spherical parameterization:

Conjecture 3 Let $\mathcal{M}$ be a genus zero surface in $\mathbb{R}^{3}$. Let $\Phi_{1}, \Phi_{2}$ and $\Phi_{3}$ be three orthogonal eigenfunctions of the Laplace-Beltrami operator. We assume they have only two nodal domains. Then the mapping

$$
\begin{aligned}
\Phi: \mathcal{M} & \longrightarrow \mathbb{S}^{2} \\
p & \longmapsto\left(\sqrt{\Phi_{1}(p)^{2}+\Phi_{2}(p)^{2}+\Phi_{3}(p)^{2}}\right)^{-1}\left(\Phi_{1}(p), \Phi_{2}(p), \Phi_{3}(p)\right)
\end{aligned}
$$

is well defined and it is a $C^{\infty}$ diffeomorphism. 
Remark 2. The orthogonality condition is more general than assuming different eigenvalues and allows applying the conjecture on the sphere itself. In that case $\boldsymbol{\Phi}$ is exactly the identity (up to a multiplicative constant). Namely, given a point $p$ and its spherical coordinates $p=(\sin \theta \cos \phi, \sin \theta \sin \phi, \cos \theta)$, a choice of three first normalized eigenfunctions is $\sqrt{\frac{3}{4 \pi}} \cos \theta, \sqrt{\frac{3}{4 \pi}} \sin \theta \cos \phi$ and $\sqrt{\frac{3}{4 \pi}} \sin \theta \sin \phi$.

Remark 3. The mapping $\boldsymbol{\Phi}$ is a particular case of the mapping $\boldsymbol{\Phi}_{\lambda}: \mathcal{M} \rightarrow \mathbb{S}^{N(\lambda)}$ proposed by Pierre Bérard in [3] where $N(\lambda)$ is the number of eigenvalues inferior to $\lambda$. But it is important to note that the proper definition of $\boldsymbol{\Phi}$ is easier in [3] when $\lambda$ is large enough because the denominator is guaranteed to never vanish. It was also shown that $\boldsymbol{\Phi}_{\lambda}$ is an embedding for $\lambda$ large enough. In our case the restrictions on the topology of the eigenfunctions could guarantee the diffeomorphic aspect for only 3 eigenfunctions.

Remark 4. We first proposed a sketch of proof for the injectivity of $\boldsymbol{\Phi}$ by using properties of the mapping $\mathbf{F}: p \rightarrow\left(\Phi_{1}(p), \Phi_{2}(p), \Phi_{3}(p)\right)$. In particular, we used the formula $\Delta \mathbf{F}(p)=2 H(p) \mathbf{N}(p)$ linking laplacian of coordinates and mean curvature for hypersurfaces, combined with $\Delta \mathbf{F}=\left(-\lambda_{1} \Phi_{1},-\lambda_{2} \Phi_{2},-\lambda_{3} \Phi_{3}\right)$. But it is important to see that the first formula holds if $\Delta$ is the Laplace-Beltrami operator of $\mathbf{F}(\mathcal{M})$ (provided it is a submanifold !) which makes the second equation not true anymore.

Nevertheless our initial flaw was at the origin of experimental observations that yield the conjecture:

Conjecture 4 With the previous notations and hypotheses, $\mathbf{F}(\mathcal{M})$ is a genuszero surface whose mean curvature has a constant sign.

\section{Preliminary results}

Our initial strategy to tackle the first conjecture was:

- to prove first that intersection points of two nodal sets exist, thanks to global arguments.

- to characterize those intersections in terms of the angle between the two isolines (equivalently the gradient of the eigenfunctions), by using local results on eigenfunctions. That sort of results are known for auto-intersection of nodal sets.

Those two first steps would allow a correct definition of the mapping $\boldsymbol{\Phi}$ but the diffeomorphic aspect remains the most difficult part.

Proposition 1 Let $\mathcal{M}$ be a genus zero surface in $\mathbb{R}^{3}$. We consider two eigenfunctions $\Phi$ and $\Psi$ with only two nodal domains and different associated eigenvalues. Then their nodal sets have at least one intersection point. 
Proof. Let $\lambda$ and $\lambda^{\prime}$ be the two eigenvalues associated to $\Phi$ and $\Psi$. Since $\Phi$ and $\Psi$ have two nodal domains, their nodal sets divide $\mathcal{M}$ in two parts respectively. If we assume that $N(\Phi) \cap N(\Psi)=\emptyset$, we have a partition of $\mathcal{M}$ in three connected domains $D_{1}, D_{2}, D_{3}$ and the two nodal sets (of measure 0 ).

where

$$
\begin{aligned}
& D_{1}=\{p \mid \Phi(p)>0, \Psi(p)>0\} \\
& D_{2}=\{p \mid \Phi(p)>0, \Psi(p)<0\} \\
& D_{3}=\{p \mid \Phi(p)<0, \Psi(p)<0\}
\end{aligned}
$$

The figure on the right provides a schematic illustration of the configuration. Inspired by this figure, we define $B=D_{2}$ and use the Green formula in two different ways:

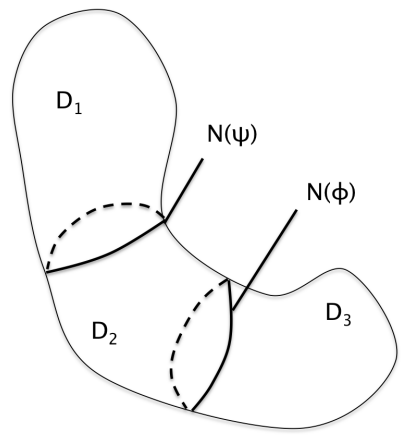

$$
\begin{aligned}
\lambda \int_{B} \Phi \Psi & =\int_{B}-\Psi \Delta \Phi=\int_{B} \nabla \Psi \cdot \nabla \Phi-\int_{\partial B} \Phi(\nabla \Psi \cdot \mathbf{n}) \\
\lambda^{\prime} \int_{B} \Phi \Psi & =\int_{B}-\Phi \Delta \Psi=\int_{B} \nabla \Psi \cdot \nabla \Phi-\int_{\partial B} \Psi(\nabla \Phi \cdot \mathbf{n})
\end{aligned}
$$

to obtain

$$
\left(\lambda^{\prime}-\lambda\right) \int_{B} \Phi \Psi=\int_{\partial B} \Phi(\nabla \Psi \cdot \mathbf{n})-\int_{\partial B} \Psi(\nabla \Phi \cdot \mathbf{n})
$$

The boundary $\partial B$ equals $N(\Phi) \cup N(\Psi)$. On $N(\Phi)$ (resp. $N(\Psi)$ ) we have $\Phi=0$ (resp. $\Psi=0)$ and on $N(\Psi)($ resp. $N(\Phi))$ we have also $\nabla \Psi \cdot \mathbf{n}=0(\operatorname{resp} . \nabla \Phi \cdot \mathbf{n}=0)$ since $N(\Psi)$ is a level set of $\Psi$ (resp. $\Phi$ ). Then the two integrals in the right term of (4) vanish which leads to $\int_{B} \Phi \Psi=0$. It is a contradiction since both $\Phi$ and $\Psi$ have a constant sign on $B$.

Remark 5. We can find simple examples where this proposition fails when the nodal domains are more than 2. For very elongated ellipsoids the second and third eigenfunctions have respectively 3 and 4 nodal domains. Numerical simulations (not shown here) revealed that the three first eigenfunctions have isolines that are all parallel and nodal sets have no intersection points.

Remark 6. It is probably harder to find examples of surfaces for which there is only one intersection point. This singular configuration implies a colinearity of $\nabla \Phi$ and $\nabla \Psi$ at the crossing point. Conversely if there is no colinearity at an intersection point, one can intuitively conclude that there is at least a second intersection point by an argument à la Jordan.

Next our initial attempt to characterize the local behavior at the intersection point followed ideas exposed in [4. In particular Theorem 2.5 says that when the nodal lines of a given eigenfunction meet, they form an equiangular system. The 
proof relies on local approximations of solutions of elliptic partial differential equations close to the origin in the $C^{\infty}$ case thanks to a theorem by Lipman Bers. Nevertheless this strategy appeared to be too general in our case and we were not able to obtain a relationship linking angle between nodal sets of two eigenfunctions and other quantities such as the local mean curvature, even if numerical computations may reveal interesting behaviors (see Fig 3 right).

\section{Experimental results}

Data We implemented our approach on 138 triangular meshes of cortical surfaces from the OASIS database that were segmented through FreeSurfer software. The number of vertices ranges from 106914 to 167230 vertices. Laplace-Beltrami eigenfunctions were computed through a variational formulation of Equation (2) and a discretization with Finite Element Methods [6]. On Fig 1 we displayed the three first eigenfunctions and the nodal set in green for a given surface. We observed that the three first eigenfunctions had only 2 nodal domains (yellow and blue in each case). The codes were implemented in MATLAB on a Mac with a $2.6 \mathrm{GHZ}$ processor. CPU time ranges from $3.76 \mathrm{~s}$ to $6.71 \mathrm{~s}$.

Diffeomorphic aspects On Fig 2 we illustrated our approach for a brain mesh at left on which the mean curvature was computed following 6 and was represented as an image. The surface $\mathbf{F}(\mathcal{M})$ was represented on the middle with the initial curvature. The orientation followed the one of the brain at left. On the right we showed the sphere $\mathbf{\Phi}(\mathcal{M})$ and the inital mean curvature with the same orientation as previously.

Next we verified that for all the meshes, the number of nodal domains of the three first non-trivial eigenfunctions were exactly 2 . We evaluated the number of flipped faces in percentage, ranging from $0.008 \%$ to $7.01 \%$ (average: $0.29 \pm 0.7 \%$ ). Fig 4 left showed an example of such faces and we observed that they correspond to local defects in the mesh, probably affecting a proper approximation of eigenfunctions.
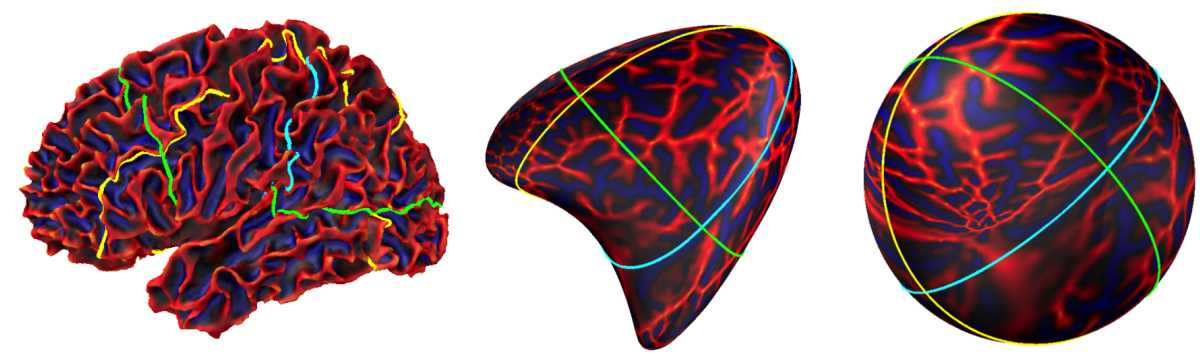

Fig. 2: Left: Cortical surface $\mathcal{M}$ and its mean curvature. Colormap goes from blue (negative) to red/yellow (positive). Middle: $\mathbf{F}(\mathcal{M})$. Right: spherical surface with the initial curvature. 
Reproducibility On Fig 3 left and middle we represented the intersection points of the 3 first nodal sets for all the meshes superimposed in the Talairach space, a classical reference system in neuroimaging. We observed first that those intersection points are only 6 for each brain and secondly that they are consistently distributed in $3 \mathrm{D}$. This result is of course related to the stability of the first eigenfunctions across the different brains and is more directly interpretable by looking at points than functions. On Fig 3 right we displayed the distribution of angles between nodal sets. The distribution is unimodal in one case (intersection of $\Phi_{1}$ and $\Phi_{3}$ ) and bimodal for the other cases.

Distorsions Finally we obtained evaluations of geometric distorsions through angular errors and relative error on lengths across all the meshes (Fig 4 middle and right). Those errors are discrepencies between the values measured on the initial mesh and on the spherical representation. Even if the errors are larger than for methods that explicitly minimize distorsions [5]1, the average values are of the same order of magnitude.
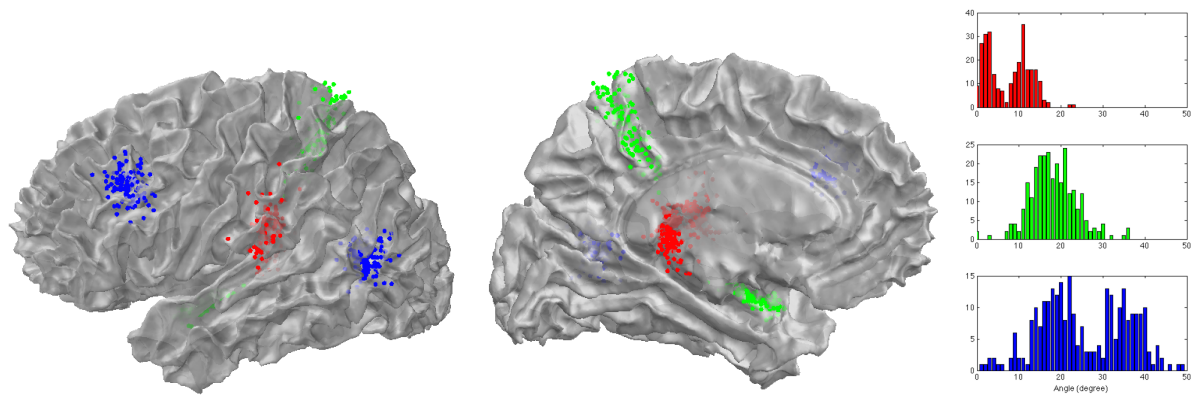

Fig. 3: Left and middle: The 6 intersection points of the 3 first nodal sets for all the meshes superimposed in the Talairach space. A slightly transparent cortical mesh is shown to illustrate the reproducibility. Right: Distribution of angles between nodal sets of 1st and 2nd, 1st and 3rd, 2nd and 3rd eigenfunctions. On the three figures the colors are matched.

\section{Discussion and perspectives}

In this work we have proposed a spherical mapping with preliminary mathematical and empirical results in favor of a $C^{\infty}$ diffeomorphic property. Cortical surfaces were very suitable in our case because they satisfy the property proposed in our mathematical part, with a number of nodal domains equal to 2 . In practice we obtained (a limited number of) flipped faces due to mesh irregularities. Moreover the computation time was $5 \mathrm{~s}$ in average, which outperforms the recent approach in [8]. Our approach offers a correct stability across a group of 
brain shapes, in the sense that interesting features such as intersection of nodal sets are quite consistent. Our mapping is probably suboptimal when it comes to match brain meshes with respect to strategies using more eigenfunctions [10]. Nevertheless our method would be very appropriate for a faster initialization of a spherical mapping instead of a Gauss map and Tutte map [7. It can also be used "on the fly" for extensive simulations [9].

We could try to generalize our results for any kind of spherical mesh by looking for eigenfunctions associated to larger eigenvalues in the spectrum, with only 2 nodal domains. But there is no guarantee that there exist 3 eigenfunctions of this kind. We could think also to possible extensions for manifolds of higher dimension. In [2] results have been obtained to bound the embedding dimension of Laplacian eigenfunctions map thanks to Ricci curvature and injectivity radius. A topological condition on the number of nodal domains could produce complementary views on this question.
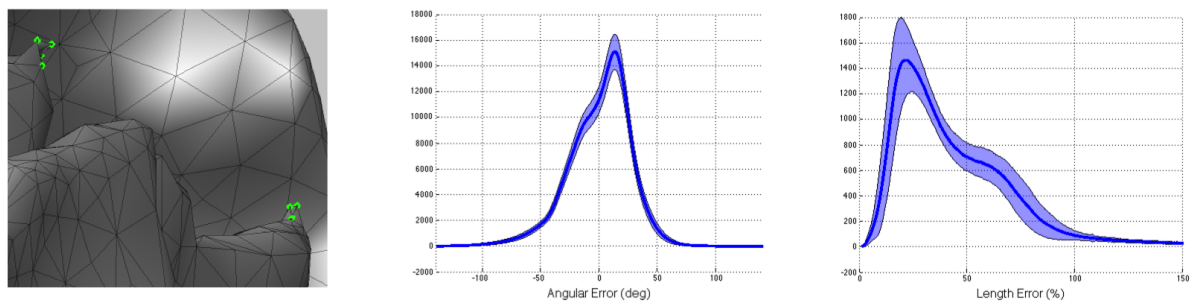

Fig. 4: From left to right: Examples of flipped triangles in green, distributions of angular error (in degree) and relative error on distances (\%).

\section{Aknowledgments}

We would like to thank the reviewers for their very constructive comments. In particular a careful observation by one the reviewer is at the origin of the remark 4 and of important modifications in the structure of the manuscript.

\section{References}

1. G. Auzias, J. Lefevre, A. Le Troter, C. Fischer, M. Perrot, J. Régis, and O. Coulon. Model-driven harmonic parameterization of the cortical surface: Hip-hop. IEEE Trans. Med. Imaging, 32(5):873-887, 2013.

2. J. Bates. The embedding dimension of laplacian eigenfunction maps. Applied and Computational Harmonic Analysis, 37(3):516-530, 2014.

3. P. Bérard. Volume des ensembles nodaux des fonctions propres du laplacien. Séminaire de théorie spectrale et géométrie, 3:1-9, 1984.

4. S.-Y. Cheng. Eigenfunctions and nodal sets. Commentarii Mathematici Helvetici, 51(1):43-55, 1976. 
5. B. Fischl, M. I. Sereno, and A. M. Dale. Cortical surface-based analysis: Ii: Inflation, flattening, and a surface-based coordinate system. Neuroimage, 9(2):195-207, 1999.

6. D. Germanaud, J. Lefèvre, R. Toro, C. Fischer, J. Dubois, L. Hertz-Pannier, and J.-F. Mangin. Larger is twistier: Spectral analysis of gyrification (spangy) applied to adult brain size polymorphism. NeuroImage, 63(3):1257-1272, 2012.

7. X. Gu, Y. Wang, T.F. Chan, P.M. Thompson, and S.T. Yau. Genus zero surface conformal mapping and its application to brain surface mapping. Medical Imaging, IEEE Transactions on, 23(8):949-958, 2004.

8. R. Lai, Z. Wen, W. Yin, X. Gu, and L. M. Lui. Folding-free global conformal mapping for genus-0 surfaces by harmonic energy minimization. Journal of Scientific Computing, 58(3):705-725, 2014.

9. J. Lefevre, V. Intwali, L. Hertz-Pannier, P. S. Hüppi, J.-F. Mangin, J. Dubois, and D. Germanaud. Surface smoothing: a way back in early brain morphogenesis. In Medical Image Computing and Computer-Assisted Intervention-MICCAI 2013, pages 590-597. Springer, 2013.

10. Herve Lombaert, Jon Sporring, and Kaleem Siddiqi. Diffeomorphic spectral matching of cortical surfaces. In Information Processing in Medical Imaging, pages 376389. Springer, 2013.

11. A. Sheffer, E. Praun, and K. Rose. Mesh parameterization methods and their applications. Foundations and Trends® in Computer Graphics and Vision, 2(2):105$171,2006$.

12. S. Zelditch. Local and global analysis of eigenfunctions. arXiv preprint arXiv:0903.3420, 2009. 